\title{
Comprehensive cytotoxicity analysis of polysaccharide hydrogel modified with cerium oxide nanoparticles for wound healing application
}

\author{
A. L. Popov ${ }^{1,2}$, V. V. Andreeva ${ }^{3}$, N. V. Khohlov ${ }^{4}$, K. A. Kamenskikh ${ }^{1}$, V. B. Gavrilyuk ${ }^{1}$, V. K. Ivanov ${ }^{2}$ \\ ${ }^{1}$ Institute of Theoretical and Experimental Biophysics of the Russian Academy of Sciences, \\ Institutskaya str., 3, Pushchino, 142290, Russia \\ ${ }^{2}$ Kurnakov Institute of General and Inorganic Chemistry of the Russian Academy of Sciences, \\ Leninskiy prosp., 31, Moscow, 119991, Russia \\ ${ }^{3}$ Moscow Regional Research and Clinical Institute, Laboratory of Medical and Physics Research, \\ Shchepkina str. 61/2, Moscow, 129110, Russia \\ ${ }^{4}$ I. M. Sechenov First Moscow State Medical University, Bolshaya Pirogovskaya str., 19s1, \\ Moscow, 119146, Russia \\ antonpopovleonid@gmail.com, viktoriaa@yandex.ru, nikolay.khokhlov@gmail.com, \\ kristina.kamensk@mail.ru,vbg@mail.ru,van@igic.ras.ru
}

PACS 68.65.-k, 81.20.-n, 82.70.Dd, 87.80.-y

DOI 10.17586/2220-8054-2021-12-3-329-335

\begin{abstract}
This paper is aimed at the experimental study (in animal models) of acute toxicity and irritating properties of polysaccharide hydrogel modified with cerium oxide nanoparticles. In the acute experiment, there were no indications of irritating action of the gel at the site of application when the hydrogel was administered intragastrically or epicutaneously. No lethal effects were registered during this experiment even at the highest concentration. The results obtained demonstrate the lack of acute toxicity and local irritability of the synthesized hybrid hydrogel, which allows to classify the developed hybrid hydrogel as the relatively low-risk drug.
\end{abstract}

Keywords: wound healing, hydrogel, cerium oxide nanoparticles, epicutaneous application, regeneration, cell proliferation.

Received: 13 May 2021

\section{Introduction}

Nanocrystalline cerium oxide has recently been proven to be a promising inorganic antioxidant and a key component of a wide range of biomedical products [1-4]. Its unique redox activity, high biocompatibility and low toxicity make it very different from many other nanomaterials [5-7]. The ability of $\mathrm{CeO}_{2}$ nanoparticles to switch between $\mathrm{Ce}^{3+}$ and $\mathrm{Ce}^{4+}$ states allows them to inactivate a wide range of reactive oxygen species (ROS) and free radicals [8]. Previously, it has been shown that $\mathrm{CeO}_{2}$ nanoparticles are capable of mimicking the activity of a number of endogenous enzymes, such as catalases and superoxide dismutases (SOD) [9]. The most significant feature of $\mathrm{CeO}_{2}$ nanoparticles is their ability to recover their activity and to participate in redox reactions multiple times, thus making this compound one of the most advantageous nanomaterials in biomedical applications. Meanwhile, the catalytic activity of $\mathrm{CeO}_{2}$ nanoparticles and their role (pro- or antioxidant) strongly depend on the nanoparticle's microenvironment [10-13]. Previously, it has been shown that $\mathrm{CeO}_{2}$ nanoparticles under acidic conditions exhibit pro-oxidant properties, whereas in neutral or weakly alkaline media, they are strictly antioxidant [14]. Also, it has been shown that the catalytic activity of $\mathrm{CeO}_{2}$ nanoparticles depends on the concentration of trivalent ions on the nanoparticle's surface; in this way, SOD-mimetic activity of $\mathrm{CeO}_{2}$ increases with the increase in $\mathrm{Ce}^{3+}$ concentration [15]. Therefore, the microenvironment affects directly the physicochemical characteristics of $\mathrm{CeO}_{2}$ nanoparticles thus controlling their catalytic and biological activity.

In this study, biocompatible citrate-stabilized $\mathrm{CeO}_{2}$ nanoparticles were synthesized and were integrated into a polysaccharide hydrogel matrix for use as a wound healing agent. The choice of hydrogel matrix and the filler was based on the existing data on $\mathrm{CeO}_{2}$ effect on the skin regeneration process. In particular, $\mathrm{CeO}_{2}$ nanoparticles possess striking anti-inflammatory activity [6], affect immune cells [16], that could significantly shorten the Phase 2 of the regeneration process (inflammation phase), and thus accelerate the start of the Phase 3 - the proliferative phase of the regeneration process. As it has previously been demonstrated, $\mathrm{CeO}_{2}$ nanoparticles are capable of accelerating the proliferation of fibroblasts, mesenchymal stem cells and osteoblasts $[3,17,18]$. It has also been previously shown that the integration of nanoparticles into various biopolymers [19] and scaffolds provides enhanced cell proliferation [20]. An additional advantage of using cerium oxide nanoparticles in the structure of wound-healing hydrogel is that they possess bacteriostatic [21] and bactericidal [22] action as well as antiviral activity [23]. Such multifaceted 
biological activities provides an opportunity for cerium oxide nanoparticles to serve as a unique bioactive component in biomedical products design and development.

The polysaccharide hydrogel matrix allows one to not only provide a convenient application of the preparation onto the skin, but is also capable of providing prolonged release of nanoparticles from the matrix into the lesion, reducing inflammation and staving off infection, as well as stimulating proliferative fibroblast activity.

\section{Materials and methods}

\subsection{Hydrogel synthesis}

The hydrogels were made from citrate-stabilized cerium oxide nanoparticles prepared according to the previously reported protocol [24], using pectin, fucoidan, sodium alginate, water-soluble derivatives of cellulose (carboxymethyl cellulose) and purified water. Briefly, $1.0 \mathrm{~g}$ fucoidan, $1.0 \mathrm{~g}$ sodium alginate, $2.0 \mathrm{~g}$ pectin, $8.0 \mathrm{~g}$ carboxymethyl cellulose were dissolved in $100 \mathrm{ml}$ distilled water. The mixture was mixed at $25^{\circ} \mathrm{C}$ and then left for $96 \mathrm{~h}$ at $4{ }^{\circ} \mathrm{C}$ until the formation of a homogeneous gel. Then, $100 \mathrm{ml}$ of the $\mathrm{CeO}_{2}$ sol $\left(2-4 \mathrm{~nm} \mathrm{CeO} \mathrm{C}_{2}\right.$ nanoparticles; $10^{-2} \mathrm{M} \mathrm{CeO}_{2}$ concentration) was added and the mixture was vigorously homogenized. Before application, the gel was sterilized in an autoclave at $120^{\circ} \mathrm{C}$.

\subsection{Laboratory animals}

Experimental procedures were performed on Wistar white rats and outbred white mice of both genders. Rats and mice came from the breeding nursery of laboratory animals "Andreevka", Moscow region. Rats weighing 180-190 g at the age of 12-13 weeks and mice weighting 18-20 $\mathrm{g}$ at the age of 8-9 weeks were used.

Animal maintenance was carried out in accordance with the rules adopted by the European Convention for the Protection of Vertebrate Animals (Strasbourg, 1986). Rats and mice were kept in plastic cages, 10 individuals per each cage. The animals were fed twice a day. All-in-one feed for laboratory animals (PK-120-1) produced by Laboratorsnab LLC was filled ad libitum into the feed hopper at the top of the cage. Animals had unlimited access to water using special drinking bottles for rodents. Bacteriological analysis and sanitary chemical examination of water were performed initially. There was no contamination of the bedding, feed and water that could affect the results of the study.

\subsection{Analysis of the effect ceria-containing polysaccharide hydrogel upon epicutaneous application}

To study the effect of polysaccharide hydrogel modified with cerium oxide nanoparticles upon epicutaneous application, a day prior to the experiment, the square of $2.5 \times 2.5 \mathrm{~cm}$ was depilated by trimming (plucking) of animal's dorsum. The hydrogel was preheated in a water bath to $38-40^{\circ} \mathrm{C}$ and then applied with a glass rod onto depilated area of rat's dorsum at ascending doses according to Litchfield-Wilcoxon method. Similar doses of petroleum jelly were administered to control animals in the same way. Thereafter, the animals were placed for 6 hours into special individual plexiglass containers to restrict locomotor activity and to prevent gel licking. After releasing the rats from the containers, the excess of the gel was removed from the skin with filter paper, and animals were placed for 14 days into check cages for observation. The acute toxicity testing was carried out at the maximum possible doses of gel on up to $20 \mathrm{~g} / \mathrm{kg}$ by epicutaneous application.

\subsection{Acute toxicity testing of polysaccharide hydrogel modified with cerium oxide nanoparticles}

Acute toxicity of gels upon intragastric administration was assessed both in white rats and mice of both genders, and upon epicutaneous application in the mice only. Groups of 12 animals (6 males and 6 females) were used in the experiments. The absence of external symptoms (external manifestation of bacterial infections, physical activity, etc.) and homogeneity of groups by body weight $( \pm 10 \%)$ were considered as randomization acceptance criteria. To estimate acute toxicity characteristic values, polysaccharide hydrogel was administered intragastrically (i.g.) to white rats and mice of both genders in ascending doses (dosing accuracy is achieved by varying the volume of gel administered) according to Litchfield-Wilcoxon method. Upon oral administration, the gel was heated in a water bath to $38-40^{\circ} \mathrm{C}$ to a liquid state. An atraumatic metal gastric catheter was used for administration and it was inserted up to the stomach. Control animals were administered with similar volumes of petroleum jelly softened with warm water. Acute toxicity testing was carried out at the maximum possible doses of up to $25 \mathrm{~g} / \mathrm{kg}$ by total gel weight. The mortality rate, lethal time for animals, symptoms of poisoning were recorded for 14 days, overall systemic condition and behavior were examined daily; the weight of the animals, food and water consumption were monitored. The dead animals were subjected to autopsy and macroscopic examination. After day 14, all the animals survived were euthanized in a $\mathrm{CO}_{2}$-chamber and dissected. Hematologic studies, macroscopic description and the evaluation of mass coefficients of internal organs, histological studies were carried out. 


\subsection{List of recorded indicators of experimental animals}

The animals were weighed just prior to administration of the hydrogel, on days 2 and 7, to control body weight dynamics, and just prior to euthanasia (day 14) to calculate the percentage of organ weight to body weight. To account for water and food consumption, rats were transferred to metabolic cages. All animals treated with the highest dose of tested hydrogel were selected for pathomorphological studies. The pathomorphological study included necropsy, macroscopic study, weighing and histological examination of internal organs. Necropsy was performed under the direct supervision of a pathologist. After euthanasia, the animals were carefully examined for the presence of external pathological signs. Examination of the gastrointestinal mucous membranes allowed us to estimate the local irritating effect of tested gel. The examination of overall state of the thoracic and abdominal cavities as well as a macro- and microscopic examination of the internal organs (heart, lungs with trachea, thymus, liver, spleen, kidneys, adrenal glands, brain, testicles or ovaries) was carried out.

\subsection{The statistical evaluation of the effect}

Statistical treatment of the results was performed using the STATISTICA 8.0 software. Data were presented as the sample mean $\mathrm{M}$, the standard error of the mean $\mathrm{m}$, and the achieved level of significance $\mathrm{p}$. Lowest accepted significance level of differences was taken as $p \leq 0.05$. In some cases, especially when there was a natural drift of indicators (e.g. animal body weight gain over the observation period, etc.), the methods of dispersion analysis were used.

\section{Results and discussion}

Upon administering high doses of the gel (up to $25 \mathrm{~g} / \mathrm{kg}$ ), no mortality was noted among mice and rats. There were also no significant abnormalities in overall behavior and systemic condition of animals. The oral administration of high doses of the gel (more than $6 \mathrm{~g} / \mathrm{kg}$ ) showed limited retardation of animals, a reduction of feed intake on the first day and diarrhea on day 2 after gel administration. Similar reactions were observed in control animals that received large volumes of petroleum jelly. Limited retardation and reduction of feed intake on the first day occurred in the control group of animals, which were apparently not related to the toxic effect of polysaccharide hydrogel, but rather to the stressful effect of the oral administration procedure of large volumes. Such a discomfort was both due to mechanical troubles in the gastrointestinal tract due to large volumes of gel administered as well as to the nature of gelling agents (laxative effect could be caused by the presence of cellulose derivatives). The condition of the animals then started to normalize and after 24 hours their overall behavior and systemic condition did not differ from those in the control group and from the baseline status.

The dissection of the animals revealed a venous congestion of internal organs. There were no signs of gastrointestinal irritation. There were no gender differences during intoxication.

The results of animal body weight measurements are shown in Tables 1 and 2.

TABLE 1 . The effect of acute intragastric administration of $\mathrm{CeO}_{2}$-containing polysaccharide hydrogel on mice's body weight $(\mathrm{g}, \mathrm{M} \pm \mathrm{m})$

\begin{tabular}{|c|c|c|c|c|}
\hline \multirow{2}{*}{ Time, days } & \multicolumn{3}{|c|}{ Experimental group and gender } \\
\cline { 2 - 5 } & \multicolumn{2}{|c|}{ Control } & \multicolumn{2}{c|}{ Hydrogel } \\
\cline { 2 - 5 } & $\mathbf{M}$ & $\mathbf{F}$ & $\mathbf{M}$ & $\mathbf{F}$ \\
\hline \hline Initially & $18.7 \pm 0.4$ & $19.3 \pm 0.2$ & $19.2 \pm 0.3$ & $18.8 \pm 0.3$ \\
\hline 2-nd day & $18.4 \pm 0.3$ & $19.2 \pm 0.1$ & $19.1 \pm 0.2$ & $18.9 \pm 0.2$ \\
\hline 7-th day & $19.2 \pm 0.2$ & $20.2 \pm 0.3$ & $19.2 \pm 0.2$ & $19.3 \pm 0.4$ \\
\hline 14-th day & $20.1 \pm 0.4$ & $20.4 \pm 0.2$ & $19.9 \pm 0.1$ & $19.7 \pm 0.2$ \\
\hline
\end{tabular}

On the second day after administration, a slight decrease was observed in the average body weight of all animals treated with $\mathrm{CeO}_{2}$-containing gel. The same effect has been registered in the control group (though to a lesser extent), so it can be attributed to general stress. To exclude this effect (most likely caused by stress the associated with involvement of animals in an experimental procedure), weight changes relative to initial values were compared. The introduction of the hybrid hydrogel to animals did not lead to significant differences in the dynamics of water and food consumption (Tables 3,4). 
TABLE 2. The effect of acute intragastric administration of $\mathrm{CeO}_{2}$-containing polysaccharide hydrogel on rats' body weight $(\mathrm{g}, \mathrm{M} \pm \mathrm{m})$

\begin{tabular}{|c|c|c|c|c|}
\hline \multirow{2}{*}{ Time, days } & \multicolumn{4}{|c|}{ Experimental group and gender } \\
\cline { 2 - 5 } & \multicolumn{2}{|c|}{ Control } & \multicolumn{2}{c|}{ Hydrogel } \\
\cline { 2 - 5 } & $\mathbf{M}$ & $\mathbf{F}$ & $\mathbf{M}$ & $\mathbf{F}$ \\
\hline \hline Initially & $176.5 \pm 3.4$ & $179.1 \pm 2.4$ & $180.1 \pm 4.6$ & $181.4 \pm 3.4$ \\
\hline 2-nd day & $176.4 \pm 3.2$ & $180.2 \pm 3.0$ & $176.2 \pm 4.3$ & $179.6 \pm 3.1$ \\
\hline 7-th day & $187.1 \pm 2.9$ & $186.6 \pm 2.2$ & $185.0 \pm 2.9$ & $183.1 \pm 3.5$ \\
\hline 14-th day & $196.3 \pm 3.1$ & $198.5 \pm 3.3$ & $195.8 \pm 4.8$ & $194.7 \pm 2.4$ \\
\hline
\end{tabular}

TABLE 3. The effect of acute intragastric administration of $\mathrm{CeO}_{2}$-containing polysaccharide hydrogel on water consumption dynamics $(\mathrm{ml} /$ day, $\mathrm{M} \pm \mathrm{m})$ by rats

\begin{tabular}{|c|c|c|c|c|}
\hline \multirow{2}{*}{ Time, days } & \multicolumn{3}{|c|}{ Experimental group and gender } \\
\cline { 2 - 5 } & \multicolumn{2}{|c|}{ Control } & \multicolumn{2}{c|}{ Hydrogel } \\
\cline { 2 - 5 } & $\mathbf{M}$ & $\mathbf{F}$ & $\mathbf{M}$ & $\mathbf{F}$ \\
\hline \hline Initially & $18 \pm 0.6$ & $15 \pm 0.7$ & $19 \pm 0.6$ & $16 \pm 0.6$ \\
\hline 2-nd day & $17 \pm 0.5$ & $16 \pm 0.4$ & $18 \pm 0.5$ & $17 \pm 0.5$ \\
\hline 7-th day & $18 \pm 0.8$ & $18 \pm 0.5$ & $20 \pm 0.3$ & $19 \pm 0.6$ \\
\hline 14-th day & $19 \pm 0.5$ & $19 \pm 0.6$ & $19 \pm 0.4$ & $18 \pm 0.7$ \\
\hline
\end{tabular}

TABLE 4. The effect of acute intragastric administration of $\mathrm{CeO}_{2}$-containing polysaccharide hydrogel on feed consumption dynamics $(\mathrm{g} / \mathrm{day}, \mathrm{M} \pm \mathrm{m})$ by rats

\begin{tabular}{|c|c|c|c|c|}
\hline \multirow{2}{*}{ Time, days } & \multicolumn{4}{|c|}{ Experimental group and gender } \\
\cline { 2 - 5 } & $\mathbf{2}$ Control & $\mathbf{F}$ & $\mathbf{M}$ & $\mathbf{F}$ \\
\cline { 2 - 5 } & $\mathbf{M}$ & $20.3 \pm 0.9$ & $20.2 \pm 0.9$ & $20.6 \pm 0.8$ \\
\hline \hline Initially & $20.5 \pm 0.9$ & $16.2 \pm 1.0 *$ & $15.9 \pm 1.3 *$ & $17.1 \pm 1.4 *$ \\
\hline 2-nd day & $15.6 \pm 1.1 *$ & 16.9 & $22.1 \pm 2.0$ \\
\hline 7-th day & $19.9 \pm 1.6$ & $24.2 \pm 1.9$ & $23.9 \pm 2.3$ & $22.6 \pm 1.8$ \\
\hline 14-th day & $21.3 \pm 2.2$ & $21.9 \pm 1.5$ & $22.0 \pm 2.8$ & 22.09 \\
\hline
\end{tabular}

Data analysis did not reveal any significant differences in body weight dynamics between the test and control animals. Slightly higher body weight gains in test groups compared with the control at the final stage of the study were not statistically significant.

No animal mortality was observed upon epicutaneous gel application. There were no external pathological manifestations that could be treated as the signs of intoxication. The condition and behavior of the test animals throughout the observation period were not different from that of the control groups, and, in all the groups, were consistent with that of intact animals. The dynamics of animal body weight in all test groups also did not differ from control (Table 5).

At the final stage of the experiment (dissection stage on the $14^{\text {th }}$ day), there were no differences between the animals treated with $\mathrm{CeO}_{2}$-containing polysaccharide hydrogel and the control group. Macroscopic studies did not reveal any species-specific or sex-specific differences, as well as the effect of the method of administration on the state of internal organs.

According to the visual inspection, the animal fur was shiny and tidy. No bald spots were observed. There were no excretions from animals. The front and hind limbs have not been altered and no deformation of the limbs has been observed. Teeth were preserved. Visible mucous membranes were pale, shiny, and smooth. Animal nutrition 
TABLE 5. The effect of polysaccharide hydrogel on experimental animals' body weight upon application onto scarified skin $(\mathrm{g}, \mathrm{M} \pm \mathrm{m})$

\begin{tabular}{|c|c|c|c|c|}
\hline \multirow{2}{*}{ Time, days } & \multicolumn{3}{|c|}{ Experimental group and gender } \\
\cline { 2 - 5 } & $\mathbf{2}$ Control & \multicolumn{2}{c|}{ Hydrogel } \\
\cline { 2 - 5 } & $\mathbf{M}$ & $\mathbf{F}$ & $\mathbf{M}$ & $\mathbf{F}$ \\
\hline \hline Initially & $180.2 \pm 4.6$ & $178.3 \pm 2.5$ & $176.3 \pm 3.0$ & $179.6 \pm 2.4$ \\
\hline 2-nd day & $181.5 \pm 4.4$ & $179.3 \pm 3.1$ & $178.2 \pm 3.3$ & $178.1 \pm 2.0$ \\
\hline 7-th day & $189.3 \pm 4.4$ & $186.5 \pm 2.6$ & $185.0 \pm 3.3$ & $187.5 \pm 2.8$ \\
\hline 14-th day & $198.8 \pm 4.8$ & $197.9 \pm 2.5$ & $199.7 \pm 3.1$ & $198.1 \pm 2.4$ \\
\hline
\end{tabular}

was satisfactory. Examination of the thoracic and abdominal cavities did not reveal any irregularities in the internal organs. The submandibular lymph nodes and salivary glands had an oval or rounded shape, a homogeneous pinkish or yellowish color and a moderate density. The thyroid glands were tightly attached to the larynx, had the usual size and density, a pinkish-reddish color. The thymus had a triangular shape, a whitish color, and a moderately dense consistency. The size and shape of the heart was not altered. The heart muscle was brownish and dense.

The surface of the lungs had a pale pink color; the lungs collapsed when the breast was opened. The dissected tissue also had a homogeneous pale pink color. The mucous membrane of the extrapulmonary bronchi was smooth, shiny, and pale pink. The spleen had a dark cherry color, smooth surface and rather dense consistency. The pancreas was pale pink and lobular.

The size and shape of the liver did not represent any changes. The liver capsule was thin and transparent. Liver tissue had a brownish color and moderately dense consistency. The size and shape of the kidneys were not different from the control, the capsule was easily removed. The surface of the organ was smooth, had a homogeneous uniform brownish-grey color. In the section of the kidneys, the cortex and the medulla were clearly distinguished.

The shape, size and density of the adrenal glands, ovaries or testicles did not differ from the control. Cerebral meninges were thin and transparent. The brain matter had moderate density, and there were no enlargements of the brain's ventricles. Intragastric administration of the polysaccharide hydrogel modified with cerium oxide nanoparticles or petroleum jelly did not reveal visible abnormalities in the gastrointestinal tract. The esophageal mucosa was shiny, smooth, and pale in color. The size and shape of the stomach did not represent any changes. There was no hyperemia, erosion, hemorrhage, indicating the irritating effect of the gel. The duodenum lumen did not show any changes, and the intestinal mucosa was shiny, smooth, pale pink. The small intestine mucosa was pale pink, shiny, smooth as well. The colon mucosa had a slightly greyish tint and was smooth and shiny.

Epicutaneous administration of polysaccharide hydrogel modified with cerium oxide nanoparticles or petroleum jelly, did not lead to any observed local abnormalities, i.e. irritation, inflammation, hyperemia. In all the groups there was a normal process of fur restoration in the depilated areas. The application of polysaccharide hydrogel modified with cerium oxide nanoparticles onto animal skin was not accompanied by any signs of irritation. To analyze possible irritation, after placing animals into special chambers their tails for $2 / 3$ were placed into test tubes filled with a gel. One hour after the completion of the gel application, no tail skin edema was observed.

Morphologically, the skin of the tails did not show any changes. Epidermis and skin appendages were intact. Epidermis layers were clearly expressed, the basal membrane was preserved. Epithelial cells of hair follicles of outer and inner root sheaths and connective tissue bag were well expressed.

Tables 6,7 show group-averaged mass coefficients of mice's and rats' internal organs.

Analysis of the mass coefficient values did not reveal any significant differences both in the groups of animals treated with polysaccharide hydrogel modified with cerium oxide nanoparticles, and in the control group. Upon epicutaneous application of polysaccharide hydrogel modified with cerium oxide nanoparticles the mass distribution of the internal organs in the groups was consistent with the normal values for intact animals.

Thus, the use of biologically active nanomaterials for the modification of various hydrogels and scaffolds gives them new functional properties which are applicable in biomedical applications [25-27].

\section{Conclusions}

Experimental data obtained suggest that single oral administration or application of ceria-containing polysaccharide hydrogel onto scarified skin of rats and mice of both genders does not cause changes in behavior and overall 
TABLE 6. Mass coefficients of white mice's internal organs upon an acute intragastric administration of high doses of polysaccharide hydrogel modified with cerium oxide nanoparticles $(\mathrm{g} / \mathrm{kg}$ body weight)

\begin{tabular}{|c|c|c|c|c|}
\hline \multirow{2}{*}{ Organ } & \multicolumn{4}{|c|}{ Experimental group and gender } \\
\cline { 2 - 5 } & \multicolumn{2}{|c|}{ Control } & \multicolumn{2}{c|}{ Hydrogel } \\
\cline { 2 - 5 } & $\mathbf{M}$ & $\mathbf{F}$ & $\mathbf{M}$ & $\mathbf{F}$ \\
\hline \hline Heart & $3.2 \pm 0.2$ & $3.3 \pm 0.2$ & $3.5 \pm 0.1$ & $3.1 \pm 0.3$ \\
\hline Lungs with a trachea & $6.2 \pm 0.2$ & $6.4 \pm 0.1$ & $6.3 \pm 0.1$ & $6.5 \pm 0.1$ \\
\hline Thymus & $0.87 \pm 0.03$ & $0.86 \pm 0.07$ & $0.88 \pm 0.02$ & $0.87 \pm 0.06$ \\
\hline Liver & $38.1 \pm 0.7$ & $35.9 \pm 1.3$ & $39.6 \pm 2.5$ & $38.2 \pm 2.2$ \\
\hline Spleen & $2.9 \pm 0.2$ & $3.0 \pm 0.1$ & $3.3 \pm 0.1$ & $3.1 \pm 0.2$ \\
\hline Kidney (left) & $4.2 \pm 0.2$ & $4.3 \pm 0.3$ & $4.3 \pm 0.3$ & $4.5 \pm 0.1$ \\
\hline Adrenal gland & $0.17 \pm 0.01$ & $0.15 \pm 0.01$ & $0.17 \pm 0.01$ & $0.16 \pm 0.01$ \\
\hline Brain & $15.5 \pm 0.5$ & $15.6 \pm 0.4$ & $15.5 \pm 0.4$ & $15.9 \pm 0.4$ \\
\hline Testicles or ovaries & $3.6 \pm 0.2$ & $0.21 \pm 0.02$ & $3.4 \pm 0.3$ & $0.18 \pm 0.02$ \\
\hline
\end{tabular}

TABLE 7. Mass coefficients of white rats' internal organs upon an acute intragastric administration of high doses of polysaccharide hydrogel modified with cerium oxide nanoparticles $(\mathrm{g} / \mathrm{kg}$ body weight)

\begin{tabular}{|c|c|c|c|c|}
\hline \multirow{2}{*}{ Organ } & \multicolumn{4}{|c|}{ Experimental group and gender } \\
\cline { 2 - 5 } & \multicolumn{2}{|c|}{ Control } & \multicolumn{2}{c|}{ Hydrogel } \\
\cline { 2 - 5 } & $\mathbf{M}$ & $\mathbf{F}$ & $\mathbf{M}$ & $\mathbf{F}$ \\
\hline \hline Heart & $3.9 \pm 0.3$ & $3.8 \pm 0.2$ & $4.1 \pm 0.2$ & $4.0 \pm 0.2$ \\
\hline Lungs with a trachea & $7.5 \pm 0.2$ & $7.3 \pm 0.4$ & $7.4 \pm 0.1$ & $7.6 \pm 0.3$ \\
\hline Thymus & $1.39 \pm 0.11$ & $1.25 \pm 0.08$ & $1.36 \pm 0.06$ & $1.27 \pm 0.11$ \\
\hline Liver & $37.9 \pm 1.4$ & $36.7 \pm 1.9$ & $36.6 \pm 1.9$ & $35.4 \pm 1.3$ \\
\hline Spleen & $3.9 \pm 0.5$ & $3.9 \pm 0.1$ & $3.9 \pm 0.2$ & $4.1 \pm 0.3$ \\
\hline Kidney (left) & $4.4 \pm 0.1$ & $4.2 \pm 0.2$ & $4.5 \pm 0.1$ & $4.3 \pm 0.2$ \\
\hline Adrenal gland & $0.09 \pm 0.02$ & $0.08 \pm 0.01$ & $0.08 \pm 0.02$ & $0.09 \pm 0.02$ \\
\hline Brain & $8.6 \pm 0.2$ & $8.9 \pm 0.2$ & $8.9 \pm 0.2$ & $8.6 \pm 0.1$ \\
\hline Testicles or ovaries & $12.2 \pm 0.1$ & $0.26 \pm 0.02$ & $12.2 \pm 0.1$ & $0.25 \pm 0.02$ \\
\hline
\end{tabular}

systemic condition of animals, do not cause any changes in mass coefficients of organs compared with control animals, as well as do not cause any macroscopic changes in the brain, internal and endocrine organs of test animals.

In acute experiments, polysaccharide hydrogel application did not lead to irritating effects at the site of application.

Thus, our toxicology study performed 14 days after acute administration as well as necropsy data allow ranking the polysaccharide hydrogel modified with cerium oxide nanoparticles as the Schedule VI of relatively low-risk drug $\left(\mathrm{LD}_{50}>25 \mathrm{~g} / \mathrm{kg}\right.$, rats, per os) [28].

It can be concluded that a polysaccharide hydrogel modified with cerium oxide nanoparticles can be considered as a safe and effective substances for the treatment of skin damage with various etiologies [29].

\section{Acknowledgements}

The reported study was funded by the grant of the President of the Russian Federation MK-138.2020.3. Synthesis of $\mathrm{CeO}_{2}$-containing hydrogels was performed by K. Kamenskikh with the support from RFBR grant 19-34-90031. 


\section{References}

[1] Bellio P., Luzi C., Mancini A., Cracchiolo S., Passacantando M., Di Pietro L., Perilli M., Amicosante G., Santucci S., Celenza G. Cerium oxide nanoparticles as potential antibiotic adjuvant. Effects of $\mathrm{CeO}_{2}$ nanoparticles on bacterial outer membrane permeability. Biochim. Biophys. Acta Biomembr., 2018, 1860, P. 2428-2435.

[2] Popov A., Popova, N., Gould D., Shcherbakov A., Sukhorukov G., Ivanov V. Ceria nanoparticles-decorated microcapsules as a smart drug delivery/protective system: Protection of encapsulated P. pyralis luciferase. ACS Appl. Mater. Interfaces, 2018, 10(17), P. 14367-14377.

[3] Rocca A., Mattoli V., Mazzolai B., Ciofani G. Cerium Oxide Nanoparticles Inhibit Adipogenesis in Rat Mesenchymal Stem Cells: Potential Therapeutic Implications. Pharm. Res., 2014, 31, P. 2952-2962.

[4] Sridharan P., Vinothkumar G., Pratheesh P., Suresh Babu K. Biomimetic potential of cerium oxide nanoparticles in modulating the metabolic gene signature in GBM-derived cell lines. J Mater Sci., 2020, 55, P. 11622-11636.

[5] Zhou D., Fang T., Lu L.-Q., Yi L. Neuroprotective potential of cerium oxide nanoparticles for focal cerebral ischemic stroke. J. Huazhong Univ. Sci. Technol., 2016, 36, P. 480-486.

[6] Hirst S.M., Karakoti A.S., Tyler R.D., Sriranganathan N., Seal S., Reilly C.M. Anti-inflammatory properties of cerium oxide nanoparticles. Small, 2009, 5(24), P. 2848-2856.

[7] Celardo I., Pedersen J.Z., Traversa E., Ghibelli L. Pharmacological potential of cerium oxide nanoparticles. Nanoscale, 2011, 3(4), P. 14111420 .

[8] Malyukin Y., Maksimchuk P., Seminko V., Okrushko E., Spivak N. Limitations of Self-Regenerative Antioxidant Ability of Nanoceria Imposed by Oxygen Diffusion. J. Phys. Chem. C, 2018, 122(28), P. 16406-16411.

[9] Seminko V., Maksimchuk P., Grygorova G., Malyukin Y.V. Mechanism and Dynamics of Fast Redox Cycling in Cerium Oxide Nanoparticles at High Oxidant Concentration. J. Phys. Chem. C, 2021, 125(8), P. 4743-4749.

[10] Seminko V., Maksimchuk P., Grygorova G., Avrunin O., Semenets V., Klochkov V., Malyukin Y. Catalytic Decomposition of Hypochlorite Anions by Ceria Nanoparticles Visualized by Spectroscopic Techniques. J. Phys. Chem. C, 2019, 123(33), P. $20675-20681$.

[11] Klochkov V.K., Sedyh O.O., Grygorova G.V., Viagin O.G., Opolonin A.D., Malyukin Yu.V. Induction and inhibition of free radicals by the $\mathrm{GdVO}_{4}: \mathrm{Eu}^{3+}$ and $\mathrm{CeO}_{2}$ nanoparticles under X-ray irradiation. Funct. Mater, 2018, 25(2), P. 294-299.

[12] Klochkov V.K., Malyukin Yu.V., Grygorova G.V., Sedyh O.O., Kavok N.S., Seminko V.V., Semynozhenko V.P. Oxidation-reduction processes in $\mathrm{CeO}_{2-x}$ nanocrystals under UV irradiation. J. Photochem. Photobiol. A, 2018, 364, P. 282-287.

[13] Malyukin Y., Klochkov V., Maksimchuk P., Seminko V., Spivak N. Oscillations of cerium oxidation state driven by oxygen diffusion in colloidal nanoceria $\left(\mathrm{CeO}_{2-x}\right)$. Nanoscale Res. Lett., 2017, 12(1), P. 566.

[14] Alpaslan E., Yazici H., Golshan N.H., Ziemer K.S., Webster T.J. pH-Dependent Activity of Dextran-Coated Cerium Oxide Nanoparticles on Prohibiting Osteosarcoma Cell Proliferation. ACS Biomater. Sci. Eng., 2015, 1(11), P. 1096-1103.

[15] Ivanov V.K., Shcherbakov A.B., Usatenko A.V. Structure-sensitive properties and biomedical applications of nanodispersed cerium dioxide. Russ. Chem. Rev., 2009, 78(9), P. 855-871.

[16] Lord M.S., Jung M., Teoh W.Y., Gunawan C., Vassie J.A., Amal R., Whitelock J.M. Cellular uptake and reactive oxygen species modulation of cerium oxide nanoparticles in human monocyte cell line U937. Biomaterials, 2012, 33(31), P. 7915-7924.

[17] Ciofani G., Genchi G.G., Liakos I., Cappello V., Gemmi M., Athanassiou A., Mazzolai B., Mattoli V. Effects of cerium oxide nanoparticles on PC12 neuronal-like cells: Proliferation, differentiation, and dopamine secretion. Pharm Res., 2013, 30, P. $2133-2145$.

[18] Niu J., Azfer A., Rogers L.M., Wang X., Kolattukudy P.E. Cardioprotective effects of cerium oxide nanoparticles in a transgenic murine model of cardiomyopathy. Cardiovasc. Res., 2007, 73, P. 549-559.

[19] Chen S., Hou Y., Cheng G., Zhang C., Wang S., Zhang J. Cerium oxide nanoparticles protect endothelial cells from apoptosis induced by oxidative stress. Biol. Trace Elem. Res., 2013, 154, P. 156-166.

[20] Lu B., Zhu D.-Y., Yin J.-H., Xu H., Zhang C.-Q., Ke Q.-F., Gao Y.-S., Guo Y.-P. Incorporation of cerium oxide in hollow mesoporous bioglass scaffolds for enhanced bone regeneration by activating the ERK signaling pathway. Biofabrication, 2019, 11(2), P. 025012.

[21] Alpaslan E., Geilich B.M., Yazici H., Webster T.J. pH-Controlled Cerium Oxide Nanoparticle Inhibition of Both Gram-Positive and GramNegative Bacteria Growth. Sci. Rep., 2017, 7, P. 45859.

[22] Agarwal C., Aggrawal S., Dutt D., Mohanty P. Cerium oxide immobilized paper matrices for bactericidal application. Mater. Sci. Eng. B, 2018, 232-235, P. 1-7.

[23] Mohamed H.E.A., Afridi S., Khalil A.T., Ali M., Zohra T., Akhtar R., Ikram A., Shinwari Z.K., Maaza M. Promising antiviral, antimicrobial and therapeutic properties of green nanoceria. Nanomedicine, 2020, 15(5), P. 467-488.

[24] Popov A.L., Popova N.R., Selezneva I.I., Akkizov A.Y., Ivanov V.K. Cerium oxide nanoparticles stimulate proliferation of primary mouse embryonic fibroblasts in vitro. Mater. Sci. Eng. C, 2016, 68, P. 406-413.

[25] Thoniyot P., Tan M.J., Karim A.A., Young D.J., Loh X.J. Nanoparticle-Hydrogel Composites: Concept, Design, and Applications of These Promising, Multi Functional Materials. Adv. Sci., 2015, 2, P. 1400010.

[26] Dannert C., Stokke B.T., Dias R.S. Nanoparticle-Hydrogel Composites: From Molecular Interactions to Macroscopic Behavior. Polymers, 2019, 11(2), P. 275.

[27] Almjasheva O.V., Garabadzhiu A.V., Kozina Yu.V., Litvinchuk L.F., Dobritsa V.P. Biological effect of zirconium dioxide-based nanoparticles. Nanosyst. Phys. Chem. Math., 2017, 8(3), P. 391-396.

[28] Gosselin R.E., Hodge H., Smith R.P., Gleason M.N. Clinical Toxicology of Commercial Products: Acute Poisoning. Williams and Wilkins, Baltimore, 1976, $332 \mathrm{p}$.

[29] Popova N.R., Andreeva V.V., Khohlov N.V., Popov A.L., Ivanov V.K. Fabrication of $\mathrm{CeO}_{2}$ nanoparticles embedded in polysaccharide hydrogel and their application in skin wound healing. Nanosyst. Phys. Chem. Math., 2020, 11(1), P. 99-109. 